\title{
Endoventricular patch plasty for dyskinetic anteroapical left ventricular aneurysm increases systolic circumferential shortening in sheep
}

Peng Zhang, MD, PhD, a,b Julius M. Guccione, PhD, a,b Susan I. Nicholas, MD, ${ }^{a}$ Joseph C. Walker, PhD, ${ }^{\mathrm{c}}$ Philip C. Crawford, CCP, ${ }^{a}$ Amin Shamal, MD, , d Gabriel Acevedo-Bolton, PhD, ${ }^{\text {b,e }}$ Michael A. Guttman, MS, Cengizhan Ozturk, MD, PhD, ${ }^{f}$ Elliot R. McVeigh, PhD, ${ }^{f}$ David A. Saloner, PhD, ${ }^{b, e}$ Arthur W. Wallace, MD, PhD, ${ }^{b, d}$ and Mark B. Ratcliffe, $\mathrm{MD}^{\mathrm{a}, \mathrm{b}}$

See related editorial on page 844.

Supplemental material is available online.
From the Departments of Surgery, ${ }^{a}$ Bioengineering, ${ }^{\mathrm{c}}$ Anesthesia, ${ }^{\mathrm{d}}$ and Radiology, ${ }^{\mathrm{e}}$ University of California, San Francisco, Calif; the Department of Veterans Affairs Medical Center, ${ }^{\mathrm{b}}$ San Francisco, Calif; and the Laboratory of Cardiac Energetics, ${ }^{\mathrm{f}} \mathrm{Na}-$ tional Institutes of Health, National Heart, Lung and Blood Institute, Bethesda, Md.

This study was supported by National Institutes of Health grant R01-HL-77921 (Dr Guccione), Veterans Affairs Merit Review (Dr Wallace), an award from the American Heart Association (Mr Walker), and R01HL-63348 (Dr Ratcliffe).

Received for publication Oct 11, 2006; revisions received Feb 8, 2007; accepted for publication March 14, 2007.

Address for reprints: Julius M. Guccione, $\mathrm{PhD}$, Division of Surgical Services (112D), San Francisco Veterans Affairs Medical Center, 4150 Clement St, San Francisco, CA 94121 (E-mail: julius.guccione@med.va.gov).

J Thorac Cardiovasc Surg 2007;134:1017-24 $0022-5223 / \$ 32.00$

Copyright (c) 2007 by The American Association for Thoracic Surgery

doi:10.1016/j.jtcvs.2007.03.060
Objective: Endoventricular patch plasty (Dor procedure) has gained favor as a surgical treatment for heart failure associated with large anteroapical myocardial infarction. We tested the hypotheses that the Dor procedure increases systolic circumferential shortening and longitudinal shortening in noninfarcted left ventricular regions in sheep.

Methods: In 6 male Dorsett sheep, the left anterior descending coronary artery and its second diagonal branch were ligated $40 \%$ of the distance from the apex to the base. Sixteen weeks after myocardial infarction, a Dor procedure was performed with a Dacron patch that was 50\% of the infarct neck dimension. Two weeks before and 2 and 6 weeks after the Dor procedure, animals underwent magnetic resonance imaging with tissue tagging in multiple short-axis and long-axis slices. Fully three-dimensional strain analyses were performed. All 6 end-systolic strain components were compared in regions $1 \mathrm{~cm}, 2 \mathrm{~cm}, 3 \mathrm{~cm}$, and $4 \mathrm{~cm}$ below the valves, as well as in the anterior, posterior, and lateral left ventricular walls and the interventricular septum.

Results: Circumferential shortening increased from before the Dor procedure to 6 weeks after repair in nearly every left ventricular region (13/16). The greatest regional change in circumferential shortening was found in the equatorial region or $2 \mathrm{~cm}$ below the base and in the posterior wall (from 9.0\% to $18.4 \% ; P<.0001$ ). Longitudinal shortening increased 2 weeks after the Dor procedure but then returned near baseline by 6 weeks after the Dor procedure.

Conclusion: The Dor procedure significantly increases systolic circumferential shortening in nearly all noninfarcted left ventricular regions in sheep.

U nsatisfactory results obtained with standard linear aneurysm repair stimulated modifications of that operation. As a consequence, in 1989 Dor and associates ${ }^{1}$ popularized patch aneurysmorrhaphy, which is performed as follows: The infarct is incised and a purse-string suture (Fontan stitch) used to reduce the circumference of the aneurysm "neck." A patch of either autologous or synthetic material is then sewn to the edge of the aneurysm neck, thereby reducing left ventricular (LV) volume, and the septal infarct is "excluded" from LV pressure. ${ }^{1}$ Residual aneurysm wall is closed over the patch. Patch aneurysmorrhaphy, now popularly referred to as the Dor procedure, was initially performed on the dilated LV with dyskinetic anteroapical myocardial infarction (MI).

Although studies document that the Dor procedure can be performed safely, ${ }^{2,3}$ the effect of the Dor procedure per se on regional LV systolic function remains unknown. Many studies have documented an improvement in global LV systolic 


\section{Abbreviations and Acronyms \\ $\mathrm{CABG}=$ coronary artery bypass grafting \\ $\mathrm{LV} \quad=$ left ventricle(ular) \\ MI $=$ myocardial infarction \\ MR = magnetic resonance \\ MRI = magnetic resonance image(ing) \\ STICH trial $=$ Surgical Treatment for Ischemic Heart failure trial \\ SVR $\quad=$ surgical ventricular restoration}

function after surgical ventricular restoration (SVR). For instance, Tanoue and associates ${ }^{4}$ used cardiac catheterization data and a single-beat method of end-systolic elastance estimation, and Schreuder and colleagues ${ }^{5}$ used a conductance catheter to document an improvement in end-systolic elastance after the Dor procedure in humans. However, we believe strongly that the mechanical effect of SVR operations depends on how the procedure affects both endsystolic and diastolic pressure-volume relationships and how those changes combine to affect ventricular function as measured by the Starling relationship. ${ }^{6}$

The effect of the Dor procedure on postoperative function is further confounded because the Dor procedure is usually performed with concomitant coronary artery bypass grafting $(\mathrm{CABG})$ and often with mitral valve repair. ${ }^{2}$ For instance, all of Tanoue's patients ${ }^{4}$ and 7 of 9 patients in Schreuder's study ${ }^{5}$ received CABGs, and the effect of improved myocardial perfusion cannot be separated from the effect of the SVR procedure. This makes studies such as that of Ribeiro and colleagues ${ }^{3}$ and the Surgical Treatment for Ischemic Heart failure (STICH) trial, ${ }^{7}$ sponsored by the National Institutes of Health, of major importance.

Given these difficulties with human data, experimental studies in animals ${ }^{8,9}$ and mathematical simulations ${ }^{10,11}$ are critically important. The sheep model of anteroapical MI has been of particular importance. ${ }^{12}$ For instance, we ${ }^{13}$ performed Dor procedures in sheep after anteroapical (dyskinetic) infarction. The Dor procedure achieved a 29\% reduction in LV volume. End-systolic elastance and diastolic compliance shifted in parallel on the pressure-volume diagram and the Starling relationship, subsequently, was not significantly changed. The primary goal of the present study was to quantify the effect of the Dor procedure on endsystolic deformation in noninfarcted LV regions in sheep. We hypothesize that patch aneurysmorrhaphy in which the infarct "neck" is reduced by $50 \%$ will significantly increase regional myocardial strain.

\section{Materials and Methods}

Animals used in this study were treated in compliance with the "Guide for the Care and Use of Laboratory Animals" prepared by the Institute of Laboratory Animal Resources, National Research
Council, and published by the National Academy Press, revised 1996.

\section{Myocardial Infarction}

Anteroapical MIs were created in 6 adult sheep as previously described. ${ }^{12}$ In brief, castrated male Dorsett sheep were anesthetized (ketamine, $33 \mathrm{mg} / \mathrm{kg}$, intramuscularly; isoflurane maintenance, $2 \%-4 \%$ inspired) and their lungs were mechanically ventilated (tidal volume $15 \mathrm{~mL} / \mathrm{kg}$; model 309-0612-800; Ohio Medical Products, Madison, Wis). During a left thoracotomy, ligatures were placed around the left anterior descending and its second diagonal branch at a point $40 \%$ of the distance from the apex to the base and sequentially tightened, causing an anteroapical MI. When present, branches of the posterior descending artery, which also supply this region, were ligated $20 \%$ of the distance from the apex to the base. After the thoracotomy was closed, the sheep recovered from anesthesia.

\section{Dor Procedure}

Sixteen weeks after MI, a partial lower sternotomy was performed with the sheep under general anesthesia. Pericardial adhesions were divided. The right femoral artery and inferior vena cava were cannulated to establish cardiopulmonary bypass (Sarns roller blood pump; Sarns, Inc, Ann Arbor, Mich; and model 541 Medtronic Affinity NT hollow-fiber oxygenator; Medtronic, Inc, Minnianapolis, Minn). The procedure was performed with the LV warm and beating. An elliptical Dacron patch was fashioned so that the major and minor axes were $50 \%$ of the corresponding infarct neck on magnetic resonance imaging (MRI). An incision was made in the apical infarct, and the transition between infarcted aneurysm and uninfarcted myocardium was determined by inspection and palpation. The patch was positioned parallel to the septum and sutured to the line of transition with interrupted 2-0 Ethibond Excel suture (Ethicon, Inc, Somerville, NJ). All dyskinetic portions of the anterior wall and septum were excluded. The LV incision was closed in an edge-to-edge fashion with running 3-0 Prolene suture (Ethicon). Bypass was discontinued, the sternotomy was closed, and the sheep recovered from anesthesia.

\section{Data Collection}

Tagged MRIs in orthogonal short- and long-axis planes were obtained from sheep 2 weeks before, 2 weeks after, and 6 weeks after the Dor procedure. Before MRI scan, the sheep were anesthetized and intubated as described immediately above. Nonferromagnetic transducer-tipped pressure catheters (model SPC-350; Millar Instruments, Inc., Houston, Tex) were placed in the left and right ventricles via the right common carotid artery and jugular vein by fluoroscopic guidance. The sheep were then placed in a sealed plastic container and transported to the MRI scanner. Each animal was positioned on its left side in the magnet (Siemens Symphony MRI scanner, 1.5 Tesla with Quantum gradients; Siemens Medical Systems, Iselin, NJ) with its chest centered in a Helmholtz coil. Metaprolol (5 mg/kg, i.v.) and atropine $(1.0 \mathrm{mg}$, i.v.) were administered before data collection and image acquisition to decrease autonomic reflexes. All data and MRI images were obtained at the end-inspiration phase with mechanical ventilation temporarily suspended. 


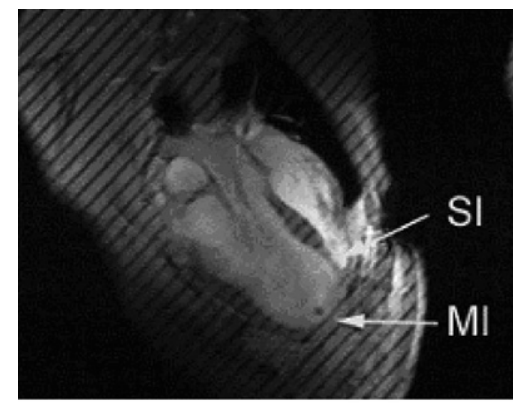

A

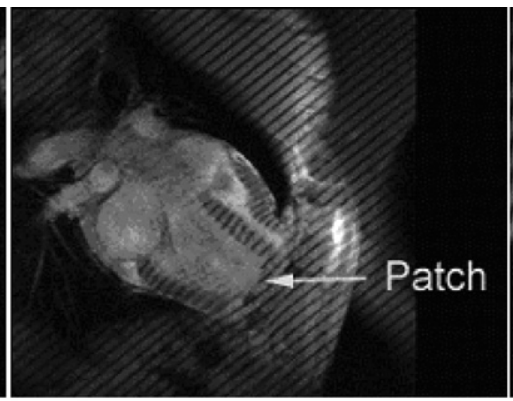

B

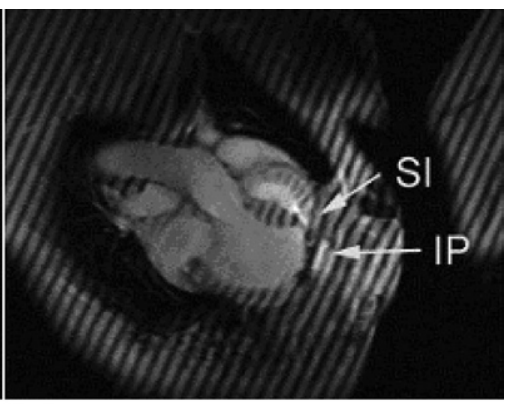

C

Figure 1. Long-axis MRIs after MI (A), 2 weeks after the Dor procedure (B), and 2 weeks after plication (C) in sheep. MI, Dykinetic myocardial infarct; $S I$, septal infarct; $I P$, infarct plication. All images were obtained at end systole. Note that the LV shapes obtained with plication and the Dor procedureare significantly different. For instance the postplication shape (C) is very globular with acute bending at the plication. The LV shape after the Dor procedure, however, seems to be rectangular, is less spherical, and is more ellipsoidal than that after plication (B).

\section{Magnetic Resonance Images}

A series of scout images was obtained to locate the heart and the true long- and short-axis planes. Subsequently, a set of 25 shortaxis imaging planes (6-mm thick) were obtained parallel to the true short-axis plane and at 5-mm intervals beginning at the level of the mitral valve and ending at a short-axis imaging plane that contained only apical myocardium and no left or right ventricular endocardium. An additional set of 6 long-axis imaging planes was obtained according to the following criteria: (1) orthogonal to the true short-axis imaging plane, (2) intersecting the centroid of the $\mathrm{LV}$, and (3) oriented in a radial fashion with $30^{\circ}$ separations between long-axis imaging planes.

Image acquisition was synchronized to the $\mathrm{R}$ wave of the electrocardiogram signal. During the actual image data acquisition, the ventilator (Hallowell 2000; Hallowell EMC, Pittsfield, Mass) was stopped for about 10 seconds at maximum inspiration (to minimize respiratory motion and the associated motion artifacts in our MRIs). During this time period, a series of images was acquired at 50-ms intervals until the approximate completion of the entire cardiac cycle. A segmented cine gradient echo sequence was used and the cardiac cycle was divided into 50-ms windows; 7 phase-encoding lines were acquired in each window. Image data were collected over 16 heart cycles providing 99 phase-encoding steps. The number of cardiac phases collected depended on the specific animal's R-R interval, with 10 cardiac phases being typical The gradient echo sequence had an echo time of $4 \mathrm{~ms}$, an excitation angle of $20^{\circ}$, and an acquisition matrix of $256 \times 102$. The field of view was set to $280 \times 230 \mathrm{~mm}^{2}$ and $280 \times 230 \mathrm{~mm}^{2}$ for the short- and long-axis images, respectively. Total magnetic resonance (MR) study time was about 45 minutes. Raw data were interpolated to $256 \times 256$ pixel images, and these were transferred to a Silicon Graphics workstation (Silicon Graphics Inc, Mountain View, Calif) and were converted from Siemens format to Silicon Graphics format with custom software (Figure 1).

\section{Data Analysis}

A slightly modified version of the MR tagging postprocessing software, "Findtags," ${ }^{14}$ was used to "contour" the endocardial and epicardial surfaces and "segment" the tags in 8 to 12 images separated by $40 \mathrm{~ms}$ for each imaging plane/slice that is fixed in space. The original number of contour points (64) in Findtags was quadrupled to account for the wide variation in LV wall thickness associated with LV aneurysm. To quantify myocardial deformation throughout the LV, we typically acquire these images in 6 different long-axis planes (at $30^{\circ}$ increments around the circumference) as well as in at least 5 different short-axis planes separated by $1 \mathrm{~cm}$. Thus, each of our 3-dimensional MR tagging studies required the Findtags postprocessing of at least 88 to 132 images. Endocardial and epicardial contours are drawn by hand (with a computer mouse) in the initial (enddiastolic) image and created automatically by Findtags in subsequent images. Findtags is not perfect, however, so these automatically created contours sometimes need to be corrected by hand. Next, the 4-dimensional B-spline-based motion tracking technique for tagged MRIs ${ }^{15}$ was used to compute all 6 unique, nonlinear, Lagrangian (Green's) strain tensor components referred to cardiac coordinates (ie, circumferential, longitudinal, and radial) at 36 locations (subendocardium, midwall, and subepicardium in each of 12 equally-spaced "sectors" around the circumference) in each short-axis plane/slice after correcting (with the postprocessed tagged long-axis MRIs) for through-plane motion. It is important to note here that these strain values are based on the displacement of the entire tag lines and not only on the displacement of tag intersection points. The "coverage" of myocardium by tag lines shown in Figure 1 is typical. The endocardial contours at end diastole and at end systole were used to compute LV volumes at these time points.

All animals were placed on their left side with the chest in the same location within the MRI scanner. Moreover, each series of scout images used to locate the heart and the true longand short-axis planes was obtained in exactly the same manner. These steps assured that each of the 12 sectors had the same anatomic location (eg, sector 8 corresponded to the middle of the anterior LV wall) in every animal and at every time point after the data analysis. We used distance of the short-axis 
imaging slice from the LV base at end diastole to define the longitudinal position in our strain comparisons.

\section{Statistical Analysis}

All values are expressed as mean \pm standard deviation and compared by repeated-measures analysis with a mixed model to test for both fixed and random effects. We performed the analysis with SAS PROC MIXED (SAS system for Windows version 9.1; SAS Institute, Inc, Cary, NC), which uses a maximum likelihood or restricted maximum likelihood estimation technique as opposed to ordinary least squares. As a consequence, subjects with missing data are not automatically deleted from the analysis. Also, variables can be either continuous or categorical and relationships between the variables can be linear or curvilinear inasmuch as the mixed model does not assume a normal distribution. ${ }^{16}$ The statistical model was as follows:

$$
\begin{aligned}
\mathrm{E}_{\mathrm{nn}, \text { Difference }}=\text { Time } & + \text { Distance }+ \text { Quadrant } \\
& +\mathrm{E}_{\mathrm{nn}, \text { Preoperative }}+\text { LVVED }+ \text { LVPES }
\end{aligned}
$$

where $\mathrm{nn}=$ strain component, Difference $=$ difference between preoperative and postoperative values, Distance $=$ distance from the LV base, Quadrant = septum, anterior wall, and so on, LVVED = volume at end diastole, and LVPES = end-systolic pressure. As can be seen, the difference between pretreatment and posttreatment strain was used as the dependent variable, and absolute pretreatment strain was used as a covariate. ${ }^{17}$ Time, distance, and quadrant cross terms were initially included, and statistically nonsignificant terms were sequentially removed beginning with those of highest order.

The statistical significance of individual group comparisons was tested with the Student $t$ test. Thus, there were 16 regions (4 quadrants at each of 4 distances), each of which received a $t$ test. With 16 regions compared, multiple-comparison corrections would have made any statistical test a prohibitively high barrier. Therefore, to keep the statistical test as sensitive as possible, we applied no multiple-comparison corrections. Statistical significance was interpreted cautiously.

\section{Results}

All 6 infarcted sheep survived the Dor procedure and completed the entire protocol. None of the sheep required inotropic support after surgery. Weight loss was minimal. Repeated-measures analysis of end-systolic LV pressures and stroke volume found no significant difference before and after the Dor procedure (Table E1). Two weeks postoperatively, the Dor procedure had a significant effect on end-diastolic pressure $(P<.0475)$ and volume $(P<.0027)$ and end-systolic volume $(P<.0260)$. Six weeks postoperatively, the Dor procedure had a significant effect on enddiastolic $(P<.0119)$ and end-systolic volume $(P<.0072)$ and ejection fraction $(P<.0058)$. There was no trend toward redilation: no significant difference in end-diastolic volume $(P=.560)$ or end-systolic volume $(P=.801)$ between 2 and 6 weeks after the Dor procedure.

The repeated-measures analysis (see Methods: Statistical Analysis) was positive with respect to end-systolic circum- ferential and longitudinal strain components $(P<.0001$ in each case). Figure 2 shows the effect of the Dor procedure on these 2 strain components. Figure 2, A, shows that circumferential shortening increases from before the Dor procedure to 6 weeks after the Dor procedure between the LV base and the border zone. Figure 2, B, shows that longitudinal shortening is increased 2 weeks after the Dor procedure near the LV base and in the border zone but then returns to baseline by 6 weeks after the Dor procedure. Figure 2, $C$ and $D$, shows the effect of the Dor procedure on circumferential and longitudinal systolic shortening when plotted against quadrant. The effect on circumferential shortening is most pronounced in lateral and posterior walls. The biggest change is from before to 6 weeks after the Dor procedure. The effect on longitudinal shortening is most pronounced in the lateral wall. There is an increase at 2 weeks but a return near baseline at 6 weeks after the Dor procedure.

Pre-Dor and 2- and 6-week post-Dor regional three-dimensional strain between end diastole and end systole are given in Tables 1 and 2. These data are presented at the centers of 4 different circumferential "quadrants" (posterior, lateral, anterior, and septal) in 4 different longitudinal "slices" $(1,2$, 3 , and $4 \mathrm{~cm}$ below the base). They can be used with our finite element method to determine myocardial contractility and stress in these LV regions after the Dor procedure.

\section{Discussion}

The primary finding of this three-dimensional MRI tagging study is that the Dor procedure significantly increases systolic circumferential shortening in all noninfarcted LV regions in sheep. The greatest regional changes in circumferential shortening are found in the equatorial region and in the posterior wall. Longitudinal shortening is increased 2 weeks after the Dor procedure but then returns near baseline by 6 weeks. Moreover, the present study (together with our previous study ${ }^{18}$ of myocyte orientation with MR diffusion tensor imaging) provides the most complete set of data to date for creating and validating mathematical models for the mechanics of the infarcted LV before and after aneurysm repair.

\section{Optimal Postoperative Shape}

We contend that no one knows the optimal LV size and shape after SVR. Certainly, if regional myocardial systolic and diastolic material properties (including the properties of the infarct) could be normal after the Dor procedure, then preinfarct normal shape would be optimal. However, the ventricle has remodeled $^{19}$ and, as documented in our prior modeling study of border zone contractility, regional systolic and diastolic myocardial material properties are altered after anteroapical MI. ${ }^{11}$ The hypothesis that our study tested was that patch aneurysmorrhaphy in which the infarct "neck" was reduced by 

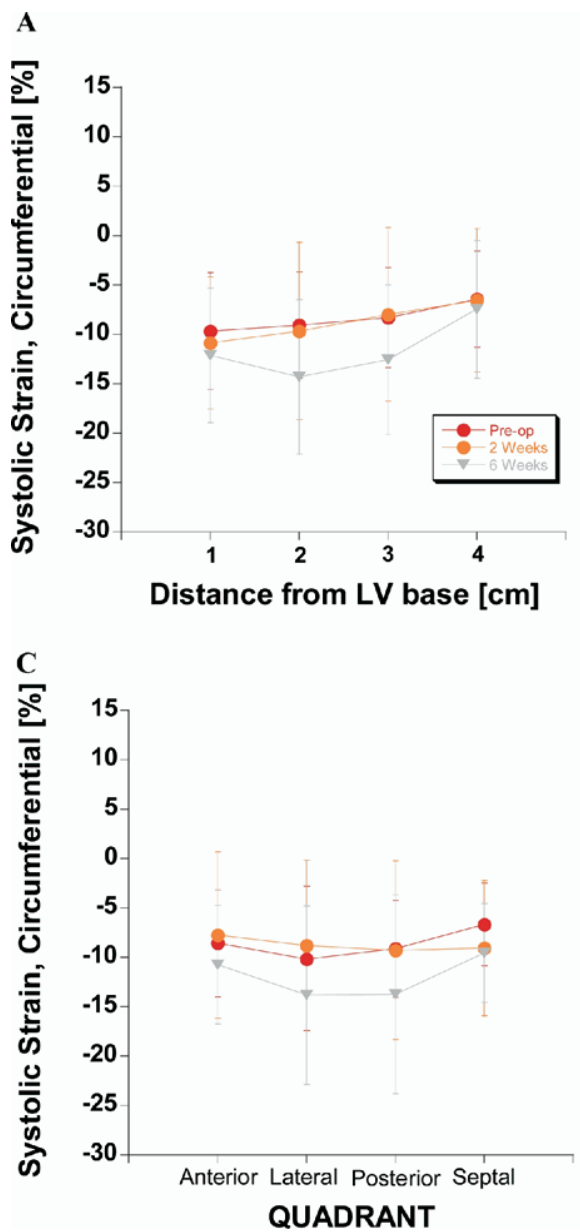

B

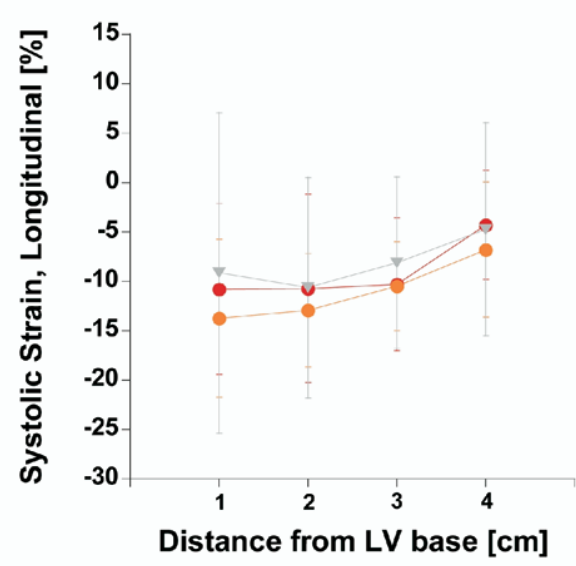

D

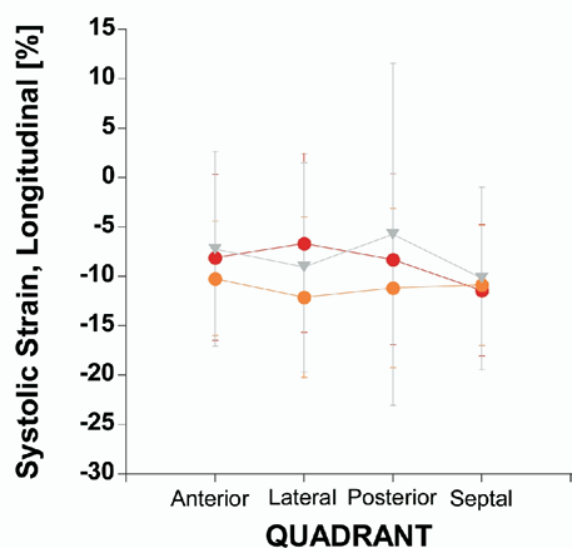

Figure 2. Effects of aneurysm plication on selected end-systolic strain tensor components. A, Circumferential strain versus distance from LV base. B, Longitudinal strain versus distance from LV base. C, Circumferential strain versus quadrant. D, Longitudinal strain versus quadrant.
$50 \%$ will increase regional myocardial strain. We did not attempt to recreate a normal shape, and it is clear that the shape we obtained is not normal. It is interesting to note that the postoperative end-diastolic volume in our study was $127 \mathrm{~mL} /$ $\mathrm{m}^{2},{ }^{20}$ and therefore larger than the size obtained with a commercially available sizer. ${ }^{21}$

\section{Stress Reduction}

The Dor procedure probably decreases the force or stress that the ventricular muscle fibers must develop to generate the preoperative end-systolic pressure or afterload. If we were to idealize LV wall geometry as a hemispherical shell that is 10 $\mathrm{mm}$ thick at end diastole and is made of an incompressible material, an analysis of our LV pressure-volume data (see Table 1 and Figure 3 in Zhang and associates ${ }^{13}$ ) based on statics $^{22}$ suggests that the Dor procedure decreases average circumferential wall stress by $25.6 \%$ at 2 weeks and by $23.1 \%$ at 6 weeks after the Dor procedure. Furthermore, mathematical modeling of the heart and cardiac operations has developed sufficiently to be of predictive value. For instance, Dang and colleagues $^{23}$ used a finite element model of the LV with an akinetic but contractile anteroapical LV wall to test the hy- pothesis that the Dor procedure increases stroke volume and reduces mean myofiber stress. They found that end-diastolic stress was reduced by $50 \%$ in the border zone and $33 \%$ in the remote myocardium. ${ }^{23}$ Additionally, end-systolic stress was reduced by $34 \%$ in the border zone and $17 \%$ in the remote myocardium. ${ }^{23}$ However, these results still must be corroborated with focused clinical data, and to date a finite element simulation of the Dor procedure with dyskinetic aneurysm has not been performed.

\section{Cause of Increased Regional Strain}

An increase in myocardial strain could be caused by an increase in preload (end-diastolic stress), a reduction in afterload (end-systolic stress), or an improvement in regional myocardial contractility. An increase in preload is unlikely. However, the reduction in end-systolic stress after the Dor procedure that is predicted by the above studies is probably sufficient by itself to increase strain. In addition, we suggest that the reduction in stress that occurs after the Dor procedure may also reverse hypertrophy and cause remote and border zone contractility to return to normal levels. In addition, although regional myofiber orientation was unchanged after linear repair in sheep, ${ }^{24}$ the 
TABLE 1. Regional circumferential strain between end diastole and end systole

\begin{tabular}{|c|c|c|c|}
\hline Region & Before Dor procedure & Two weeks after Dor procedure & Six weeks after Dor procedure \\
\hline \multicolumn{4}{|c|}{$1 \mathrm{~cm}$ below base } \\
\hline Anterior & $-9.8 \pm 5.7$ & $-10.6 \pm 6.3$ & $-14.1 \pm 6.3^{*} \dagger$ \\
\hline Lateral & $-13.8 \pm 7.7$ & $-11.6 \pm 5.3$ & $-11.5 \pm 7.9$ \\
\hline Posterior & $-8.9 \pm 6.1$ & $-10.6 \pm 8.5$ & $-13.3 \pm 8.3^{*}$ \\
\hline Septal & $-8.0 \pm 3.4$ & $-10.9 \pm 6.1^{*}$ & $-10.1 \pm 4.5^{*}$ \\
\hline \multicolumn{4}{|c|}{$2 \mathrm{~cm}$ below base (equator) } \\
\hline Anterior & $-9.9 \pm 5.0$ & $-8.3 \pm 9.5$ & $-13.1 \pm 4.3^{*} \dagger$ \\
\hline Lateral & $-12.2 \pm 7.0$ & $-10.3 \pm 8.1$ & $-16.9 \pm 8.0^{*} \dagger$ \\
\hline Posterior & $-9.0 \pm 5.1$ & $-10.6 \pm 10.0$ & $-18.4 \pm 10.8^{*} \dagger$ \\
\hline Septal & $-6.9 \pm 4.0$ & $-9.6 \pm 8.2^{*}$ & $-10.9 \pm 4.9^{*}$ \\
\hline \multicolumn{4}{|c|}{$3 \mathrm{~cm}$ below base } \\
\hline Anterior & $-8.5 \pm 5.1$ & $-6.4 \pm 9.5$ & $-10.1 \pm 4.8 \dagger$ \\
\hline Lateral & $-10.0 \pm 5.1$ & $-7.6 \pm 10.8$ & $-15.5 \pm 7.9^{*} \dagger$ \\
\hline Posterior & $-9.7 \pm 4.7$ & $-8.8 \pm 8.6$ & $-15.8 \pm 10.8^{*} \dagger$ \\
\hline Septal & $-6.2 \pm 4.6$ & $-8.7 \pm 7.0^{*}$ & $-10.5 \pm 4.1^{*}$ \\
\hline \multicolumn{4}{|c|}{$4 \mathrm{~cm}$ below base (border zone) } \\
\hline Anterior & $-6.1 \pm 5.2$ & $-5.7 \pm 7.2$ & $-5.8 \pm 4.8$ \\
\hline Lateral & $-4.6 \pm 5.9$ & $-5.7 \pm 8.3$ & $-11.5 \pm 11.1^{*} \dagger$ \\
\hline Posterior & $-9.0 \pm 3.4$ & $-7.2 \pm 8.7$ & $-7.4 \pm 6.5$ \\
\hline Septal & $-5.7 \pm 4.3$ & $-7.1 \pm 5.3^{*}$ & $-6.7 \pm 5.2$ \\
\hline
\end{tabular}

Values are mean \pm standard deviation. ${ }^{*} P<.052$ and 6 weeks after Dor procedure versus before Dor procedure. $\uparrow P<.052$ weeks versus 6 weeks after Dor procedure.

Dor procedure might align myofibers so that they contract more effectively. ${ }^{25}$

There is an improvement in longitudinal strain in some regions of the myocardium 2 weeks after repair, but this improvement is lost at 6 weeks. There are only three possible explanations for this. First, there was a temporary improvement in regional contractility that, because of regional myocyte orientation, manifested itself as an increase

TABLE 2. Regional longitudinal strain between end diastole and end systole

\begin{tabular}{|c|c|c|c|}
\hline Region & Before Dor procedure & Two weeks after Dor procedure & Six weeks after Dor procedure \\
\hline \multicolumn{4}{|c|}{$1 \mathrm{~cm}$ below base } \\
\hline Anterior & $11.5 \pm 5.4$ & $10.8 \pm 5.7$ & $-9.2 \pm 8.4$ \\
\hline Lateral & $-10.7 \pm 10.1$ & $-17.8 \pm 8.2^{*}$ & $-3.1 \pm 11.2^{*} \dagger$ \\
\hline Posterior & $-6.7 \pm 11.8$ & $-18.9 \pm 8.8^{*}$ & $-0.1 \pm 16.4^{*} \dagger$ \\
\hline Septal & $-13.4 \pm 5.6$ & $-10.1 \pm 5.5^{*}$ & $-13.0 \pm 10.1$ \\
\hline \multicolumn{4}{|c|}{$2 \mathrm{~cm}$ below base (equator) } \\
\hline Anterior & $-6.7 \pm 13.4$ & $-11.7 \pm 4.2^{*}$ & $-10.7 \pm 8.2$ \\
\hline Lateral & $-8.4 \pm 10.3$ & $-12.8 \pm 6.3$ & $-16.9 \pm 9.7^{*} \dagger$ \\
\hline Posterior & $-11.4 \pm 6.7$ & $-11.6 \pm 7.1$ & $-3.6 \pm 14.5^{*} \dagger$ \\
\hline Septal & $-14.4 \pm 5.1$ & $-15.0 \pm 4.8$ & $-12.7 \pm 7.8$ \\
\hline \multicolumn{4}{|c|}{$3 \mathrm{~cm}$ below base } \\
\hline Anterior & $-8.6 \pm 6.7$ & $-10.2 \pm 3.3^{*}$ & $-6.2 \pm 7.5 \dagger$ \\
\hline Lateral & $-6.6 \pm 6.4$ & $-8.3 \pm 6.2$ & $-9.2 \pm 4.8$ \\
\hline Posterior & $-12.0 \pm 5.6$ & $-8.9 \pm 3.5^{*}$ & $-7.4 \pm 12.7^{*}$ \\
\hline Septal & $-12.1 \pm 6.5$ & $-13.0 \pm 3.9$ & $-9.5 \pm 7.1^{*} \dagger$ \\
\hline \multicolumn{4}{|c|}{$4 \mathrm{~cm}$ below base (border zone) } \\
\hline Anterior & $-5.6 \pm 3.1$ & $-8.2 \pm 8.2^{*}$ & $-2.7 \pm 12.7 \dagger$ \\
\hline Lateral & $-1.1 \pm 5.8$ & $-9.6 \pm 8.3^{*}$ & $-7.1 \pm 10.6^{*}$ \\
\hline Posterior & $-3.0 \pm 5.7$ & $-5.3 \pm 4.9^{*}$ & $-4.0 \pm 10.1$ \\
\hline Septal & $-5.9 \pm 5.9$ & $-5.4 \pm 5.4$ & $-5.5 \pm 9.6$ \\
\hline
\end{tabular}

Values are mean \pm standard deviation. ${ }^{*} P<.052$ and 6 weeks after Dor procedure versus before Dor procedure. $\dagger P<.052$ weeks versus 6 weeks after Dor procedure. 
in longitudinal strain. However, it is not clear why an improvement in contractility would reverse. Second, the increase in strain was not due to increased contractility but to a change in load, which could be either an increase in preload or a decrease in afterload. An increase in preload could have been from too much intravascular volume in the early postoperative period, because the animals did not receive furosemide (Lasix). A change in afterload would be related to either shape change or pressure. There was a trend toward an increase in end-systolic pressure at 6 weeks. These two possibilities would require modeling to sort out. The last possibility is that there is some sort of progressive LV restraint caused by fibrosis in the pericardium.

Myocyte contractility is probably decreased in both the remote uninfarcted and border zone myocardium of the LV that has remodeled after large anteroapical MI. For instance, immediately after MI, the infarcted region loses contractile function. The remote uninfarcted myocardium compensates via the Starling relationship, end-diastolic volume is increased, and stroke volume is maintained. ${ }^{4}$ As a consequence, enddiastolic stress is increased and eccentric hypertrophy of the remote myocardium develops. For instance, Anand and colleagues ${ }^{26}$ found that myocytes isolated from the remote myocardium of rats had an increase in length of $24 \%$. Systolic function is initially preserved, but eventually contractility of the remote myocardium is decreased. ${ }^{26}$ It is unknown whether the reduction in end-diastolic stress that occurs with the Dor procedure is sufficient to reverse eccentric hypertrophy and thereby increase contractility in the remote myocardium. However, if the stress is immediately reduced by the operation and then remains unchanged until 6 weeks postoperatively whereas the strain only improves (increases) in the interval between 2 and 6 weeks, then the most likely cause is an increase in contractility in the remote myocardium that occurs between 2 and 6 weeks.

Contractility in the border zone is also decreased. For instance, Jackson and associates ${ }^{19}$ described infarct extension after anteroapical MI in sheep that surprisingly occurs in the face of normal border zone blood flow. This nonischemic infarct extension is associated with an increase in end-systolic stress. ${ }^{27}$ In addition, Guccione and colleagues ${ }^{11}$ simulated the LV with anteroapical MI and showed that the mechanism underlying mechanical dysfunction in the border zone region of LV aneurysm is primarily the result of myocardial contractile dysfunction rather than increased wall stress in this region. It is also unknown whether the reduction in end-systolic stress that occurs with the Dor procedure is sufficient to reverse nonischemic infarct extension and thereby increase regional border zone contractility.

\section{Comparison Between the Dor Procedure and Linear Aneurysm Repair}

The effect of surgical ventricular remodeling on ventricular function is difficult to determine from clinical data given that the operations often include concomitant CABG and mitral valve repair. Thus, despite the strong clinical trend away from linear repair of LV aneurysm, it is not clear that a choice for patch aneurysmorrhaphy is correct. After having performed fully three-dimensional strain analyses on a very reproducible animal model of LV aneurysm before and after each of these two types of repairs, we now are in a unique position to compare their effects on regional systolic deformation. The greatest regional differences in circumferential shortening between 6 weeks after and before the Dor procedure $(5.3 \%$ more shortening $2 \mathrm{~cm}$ below the LV base; $4.6 \%$ more shortening in the posterior wall) are larger than those between 6 weeks postplication and preplication (3.9\% more shortening in the border zone; $3.0 \%$ more shortening in the anterior wall). ${ }^{28}$ This suggests that patch aneurysmorrhaphy has a more positive effect on systolic circumferential shortening than linear repair. Moreover, patch aneurysmorrhaphy is not associated with LV redilation. Patch aneurysmorrhaphy is therefore superior to aneurysm plication.

\section{Limitations}

End-systolic radial strain tends to be more variable than does circumferential strain in tagged MRIs. ${ }^{29}$ One factor in radial strain variablility is that tagging with either parallel lines or rectangular grids results in relatively sparse sampling of myocardial motion in the radial direction. For example, a 10 -mm-thick myocardium with a tag spacing of $8 \mathrm{~mm}$ yields only one or two tag lines in the radial direction, which makes the resulting measurement of radial strain sensitive to noise in the tag line position measurements and highly influenced by the deformation model. Another source of variability in both radial and longitudinal strain is the difficulty of defining the radial and longitudinal directions on the curved LV wall, particularly at the apex.

Another limitation of the present study is the limited spatial and temporal resolution associated with our MRI acquisition. In several of our experiments, we acquired tagged short-axis images that were separated by $1 \mathrm{~cm}$. Since the long-axis lengths of repaired LVs were significantly less than those of the other LVs, strain comparisons could be made only in the short-axis slices 1 to $4 \mathrm{~cm}$ below the valves. Moreover, the border zone in the anterior LV wall was contained within the latter slice, but the other border zone regions were located below it. Thus, it is not entirely accurate to label all $\mathrm{LV}$ regions in the short-axis slice $4 \mathrm{~cm}$ below the valves as border zone regions. We acquired tagged MRIs every $40 \mathrm{~ms}$ between end diastole and end systole. This temporal resolution was not sufficient to rigorously study strain during isovolumic systole and thus prevented us from confirming the measurements of Moulton and coworkers. ${ }^{30}$

No baseline imaging (ie, before infarction) was performed during the present study. This would have allowed comparison of regional differences in contractility to be 
assessed and would have permitted therapy to be compared to normal. Such analysis would better elucidate the effect of the remodeling procedure. Since the effect of the anteroapical infarct in sheep on regional 2-dimensional (circumferential) myocardial strain has already been measured, ${ }^{31}$ however, we did not think that preinfarct and immediately postinfarct data collection was critical. Conversely, our present study extends only 6 weeks after the Dor procedure and may miss later remodeling events.

\section{Conclusions and Future Directions}

The Dor procedure significantly increases systolic circumferential shortening in all noninfarcted LV regions in sheep. This is the first objective evidence (other than that suggested by Ribeiro and colleagues ${ }^{3}$ and Zhang and colleagues ${ }^{13}$ ) that the Dor procedure does anything. Moreover, these results support our hypothesis that the effect is on border zone and remote systolic function and is mediated by stress reduction. Ideally, our mathematical modeling that is currently underway will support an improvement in remote and border zone function rather than just a reduction in load.

We thank Dr Charles McCulloch, Professor and Head, Division of Biostatistics, University of California San Francisco, for his guidance on the statistical analysis. We thank Dr J. M. Guccione, Sr, for his help in the construction of the plastic container used in this study.

\section{References}

1. Dor V, Saab M, Coste P, Kornaszewska M, Montiglio F. Left ventricular aneurysm: a new surgical approach. $J$ Thorac Cardiovasc Surg. 1989;37:11-9.

2. Dor V, Di Donato M, Sabatier M, Montiglio F, Civaia F. Left ventricular reconstruction by endoventricular circular patch plasty repair: a 17-year experience. Semin Thorac Cardiovasc Surg. 2001;13: 435-47.

3. Ribeiro GCA, Antonialli F, Lopes MM, Costa CE, Albuquerque AN, Franchini KG. Left ventricular reconstruction brings benefit for patients with ischemic cardiomyopathy. J Card Failure. 2006;12:189-94.

4. Tanoue Y, Ando H, Fukumura F, Umesue M, Uchida T, Taniguchi K, et al. Ventricular energetics in endoventricular circular patch plasty for dyskinetic anterior left ventricular aneurysm. Ann Thorac Surg. 2003; 75:1205-8; discussion 1208-9

5. Schreuder JJ, Castiglioni A, Maisano F, Steendijk P, Donelli A, Baan $\mathrm{J}$, et al. Acute decrease of left ventricular mechanical dyssynchrony and improvement of contractile state and energy efficiency after left ventricular restoration. J Thorac Cardiovasc Surg. 2005;129:138-45.

6. Ratcliffe MB, Hong J, Salahieh A, Ruch S, Wallace AW. The effect of ventricular volume reduction surgery in the dilated, poorly contractile left ventricle: a simple finite element analysis. $J$ Thorac Cardiovasc Surg. 1998;116:566-77.

7. STICH Trial. 2002. Bethesda (MD): National Institutes of Health: CRISP Database; 2002.

8. Ratcliffe MB, Wallace AW, Salahieh A, Hong J, Ruch S, Hall TS Ventricular volume, chamber stiffness, and function after anteroapical aneurysm plication in the sheep. J Thorac Cardiovasc Surg. 2000;119: $115-24$.

9. Nishina T, Nishimura K, Yuasa S, Miwa S, Nomoto T, Sakakibara Y, et al. Initial effects of the left ventricular repair by plication may not last long in a rat ischemic cardiomyopathy model. Circulation. 2001; 104(Suppl 1):I241-5.

10. Artrip JH, Oz MC, Burkhoff D. Left ventricular volume reduction surgery for heart failure: a physiologic perspective. J Thorac Cardiovasc Surg. 2001;122:775-82.
11. Guccione JM, Moonly SM, Moustakidis P, Costa KD, Moulton MJ, Ratcliffe MB, et al. Mechanism underlying mechanical dysfunction in the border zone of left ventricular aneurysm: a finite element model study. Ann Thorac Surg. 2001;71:654-62.

12. Markovitz LJ, Savage EB, Ratcliffe MB, Bavaria JE, Kreiner G, Iozzo $\mathrm{RV}$, et al. Large animal model of left ventricular aneurysm. Ann Thorac Surg. 1989;48:838-45.

13. Zhang P, Guccione JM, Nicholas SI, Walker JC, Crawford PC, Shamal A, et al. Left ventricular volume and function after endoventricular patch plasty for dyskinetic anteroapical left ventricular aneurysm in sheep. J Thorac Cardiovasc Surg. 2005;130:1032-8.

14. Guttman MA, Zerhouni EA, McVeigh ER. Analysis and visualization of cardiac function from MR images. IEEE Comp Graph Appl. 1997; 17:30-8.

15. Ozturk C, McVeigh ER. Four-dimensional B-spline based motion analysis of tagged MR images: introduction and in vivo validation. Phys Med Biol. 2000;45:1683-702.

16. Krueger C, Tian L. A comparison of the general linear mixed model and repeated measures ANOVA using a dataset with multiple missing data points. Biol Res Nurs. 2004;6:151-7.

17. Wooding W. Planning pharmaceutical clinical trials: basic statistical principals-probability and mathematical statistics. New York: John Wiley; 1994. p. 34-6.

18. Walker JC, Guccione JM, Jiang Y, Zhang P, Wallace AW, Hsu EW, et al. Helical myofiber orientation after myocardial infarction and left ventricular surgical restoration in sheep. J Thorac Cardiovasc Surg. 2005; 129:382-90.

19. Jackson BM, Gorman JH, Moainie SL, Guy TS, Narula N, Narula J, et al. Extension of borderzone myocardium in postinfarction dilated cardiomyopathy. J Am Coll Cardiol. 2002;40:1160-7; discussion 1168-71.

20. Stowe CM, Good AL. Estimation of cardiac output in calves and sheep by the dye and Fick oxygen techniques. Am J Physiol. 1960;198:987-90.

21. The TR31SVR TM Surgical Ventricular Restoration System. Chase Medical Inc; Richardson (TX): 2003.

22. Fung YC. Biodynamics: circulation. New York: Springer-Verlag; 1996. Equation 4, p 24.

23. Dang AB, Guccione JM, Zhang P, Wallace AW, Gorman RC, Gorman $\mathrm{JH}$ 3rd, et al. Effect of ventricular size and patch stiffness in surgical anterior ventricular restoration: a finite element model study. Ann Thorac Surg. 2005;79:185-93.

24. Walker JC, Guccione JM, Jiang Y, Zhang P, Wallace AW, Hsu EW, et al. Helical myofiber orientation after myocardial infarction and left ventricular surgical restoration in sheep. $J$ Thorac Cardiovasc Surg. 2005;129:382-90.

25. Buckberg GD. Imaging, models, and reality: a basis for anatomicphysiologic planning. J Thorac Cardiovasc Surg. 2005;129:243-5.

26. Anand IS, Liu D, Chugh SS, Prahash AJ, Gupta S, John R, et al, Isolated myocyte contractile function is normal in postinfarct remodeled rat heart with systolic dysfunction. Circulation. 1997; 96:3974-84.

27. Walker JC, Ratcliffe MB, Zhang P, Wallace AW, Fata B, Hsu EW, et al. MRI-based finite-element analysis of left ventricular aneurysm. Am J Physiol Heart Circ Physiol. 2005;289:H692-700.

28. Guccione JM, Walker JC, Beitler JR, Moonly SM, Zhang P, Guttman MA, et al. The effect of anteroapical aneurysm plication on endsystolic three-dimensional strain in the sheep: a magnetic resonance imaging tagging study. J Thorac Cardiovasc Surg. 2006;131:579-86.

29. Denney TS Jr, Gerber BL, Yan L. Unsupervised reconstruction of a three-dimensional left ventricular strain from parallel tagged cardiac images. Magn Reson Med. 2003;49:743-54.

30. Moulton MJ, Downing SW, Creswell LL, Fishman DS, Amsterdam DM, Szabo BA, et al. Mechanical dysfunction in the border zone of an ovine model of left ventricular aneurysm. Ann Thorac Surg. 1995;60: 986-98.

31. Kramer CM, Lima JA, Reichek N, Ferrari VA, Llaneras MR, Palmon $\mathrm{LC}$, et al. Regional differences in function within noninfarcted myocardium during left ventricular remodeling. Circulation. 1993;88 $1279-88$. 
TABLE E1. Dor versus pre-Dor

\begin{tabular}{lccc}
\hline & Before Dor & Two weeks after Dor & Six weeks after Dor \\
\hline LVP at ED $(\mathrm{mm} \mathrm{Hg})$ & $8.9 \pm 5.5$ & $17.5 \pm 5.6^{*}$ & $10.5 \pm 3.9$ \\
LVP at ES $(\mathrm{mm} \mathrm{Hg})$ & $96.2 \pm 23.2$ & $105.7 \pm 20.0$ & $123.2 \pm 19.4$ \\
EDV $(\mathrm{mL})$ & $161.6 \pm 37.1$ & $104.1 \pm 29.2^{*}$ & $101.3 \pm 22.2^{*}$ \\
ESV $(\mathrm{mL})$ & $130.1 \pm 36.0$ & $68.0 \pm 29.5^{*}$ & $66.3 \pm 14.3^{*}$ \\
SV $(\mathrm{mL})$ & $31.5 \pm 9.4$ & $31.9 \pm 13.9$ & $35.0 \pm 10.8$ \\
EF $(\%)$ & $20.6 \pm 8.1$ & $33.0 \pm 11.0$ & $34.4 \pm 7.1^{*}$
\end{tabular}

Values are mean \pm standard deviation. $L V P$, Left ventricular pressure; $E D$, end diastole; $E S$, end systole; $E D V$, end-diastolic volume; $E S V$, end-systolic volume; $S V$, stoke volume; $E F$, ejection fraction. ${ }^{*} P<.05$ 2- and 6 -week plication versus preplication. 